\title{
SWAP DM: preliminary design and schematics of a DM for extreme adaptive optics
}

\author{
Teresa Kopf ${ }^{a}$, Oliver Dietzel ${ }^{b}$, Benjamin Dargatz ${ }^{c}$, Andreas Kamm $^{\mathrm{a}}$, Mathias Bach ${ }^{\mathrm{b}}$, Klaus \\ Süßmuth ${ }^{\mathrm{b}}$, Stefan Richter ${ }^{\mathrm{c}}$, Bernd Broich ${ }^{\mathrm{c}}$, Christoph Damm ${ }^{\mathrm{a}}$, Ramona Eberhardt ${ }^{\mathrm{a}}$ Claudia Reinlein $^{\mathrm{a}}$ \\ ${ }^{a}$ Fraunhofer Institute for Applied Optics and Precision Engineering, Albert-Einstein-Str. 7, 07745 \\ Jena, Germany \\ ${ }^{\mathrm{b}}$ Physik Instrumente GmbH \& Co. KG, Auf der Roemerstrasse 1, 76228 Karlsruhe, Germany \\ ${ }^{c}$ PI Ceramic GmbH, Lindenstraße, 07589 Lederhose, Deutschland
}

\begin{abstract}
The extreme adaptive optics deformable mirror as outlined in the study of EPICS for the EELT helps to detect planets much fainter than the observed star. In doing so, excellent wavefront quality ensured by a high actuator count is mandatory. A long actuator lifetime is equally important since it reduces the number of dead actuators to a minimum, which is necessary for the coronagraphy techniques.

The Fraunhofer Institute of Applied Optics in Jena and Physik Instumente GmbH teamed up to response to an ESO Call for Tender. We developed an appropriate deformable mirror (DM) concept, and are currently elaborating the breadboards to demonstrate critical technologies. The considered DM technology is based on piezoelectric stack actuators which deform a thin-shell glass substrate. As a main feature, we provide a modular solution, meaning that actuator modules may be inserted into a DM substrate. With that an exchange of actuator modules in case of actuator failure is possible that characterizes the SWAP DM for extreme adaptive optics. In order to enable a high lifetime of the DM, we will pre-stress the actuator and use a modified PICMA® actuator, which exhibits a ceramic insulation extending the lifetime. Thus, the array benefits from an improved actuator lifetime without the requirement for an additional encapsulation which would be disadvantageous for the necessity of a low actuator pitch.
\end{abstract}

In this project several laboratory breadboards will be developed to demonstrate key aspects of the deformable mirrors and their TRL level. We present the current state of the preliminary design, the schematic design and the status of the breadboards.

\section{INTRODUCTION IN REQUIREMENTS}

ESO has provided the requirements for the DM which result from their comprehensive EPICS study [1]. The comparison between the ESO requirements [2] and the capabilities of the piezoelectric stack technology proposed by the bidder team reveals the following mirror characteristics. We aim to develop a ring-shaped deformable mirror containing an active outer diameter of $450 \mathrm{~mm}$ and a passive inner diameter of $180 \mathrm{~mm}$. The required actuator pitch is $3.7 \mathrm{~mm}$, which leads to a number of 11,000 piezoelectric actuators within the aperture. The actuators are supposed to allow for bidirectional activation of $\pm 1.5 \mu \mathrm{m}$ leading to a minimum interactuator stroke of $\pm 1.2 \mu \mathrm{m}$. Also, the actuators need to be equipped with compensation for hysteresis and non-linearity. The initial failure rate of the piezoelectric stacks should be below $0,05 \%$ meaning that 5 stacks out of 11,000 stacks may fail. In case of a higher failure rate during the required 12 years of operation, the concept for the DM foresees the replaceability of actuator modules -giving the mirror its name SWAPDM.

The optical requirements describe the quality of the mirror surface. There are three major conditions given for the flatness of the mirror surface: The defocus term needs to be below $2 \mu \mathrm{m} \mathrm{PtV}$, the sum of the high order modes must be below $2 \mu \mathrm{m}$, and after the subtraction of the Zernike modes and with piezoelectric activation the remaining root-mean square (rms) error is allowed to be maximum $10 \mathrm{~nm}$. Besides, the mirror roughness must be below $1.5 \mathrm{~nm} \mathrm{rms}$. The 
reflectivity is defined to be $97 \%$ for wavelengths between $450-1000 \mathrm{~nm}$ and $98 \%$ for wavelengths between $1000-2000 \mathrm{~nm}$.

The environmental working conditions define the functional working temperature range and relative humidity range for the DM. The mirror is required to work at temperatures between $-5^{\circ} \mathrm{C}$ and $20^{\circ} \mathrm{C}$ and a humidity range of $5-80 \%$. ESO agreed to use nitrogen flushing to reduce the circumambient humidity, which may affect the lifetime of the piezoelectric actuators or the tensile strength of adhesive bonding.

\section{SCHEMATICS}

We propose the development of actuator modules with a moderate number of piezoelectric stacks that are to be integrated into the deformable mirror. Assembling 11,000 actuators from preassembled actuator modules provides several benefits; the main advantage is the exchangeability of defect actuators by their corresponding actuator modules, which may occur during a longstanding usage. Furthermore, the precisely manufactured piezo stacks are individually inspected to guarantee for the highest quality. The piezoelectric stacks are preferred to work against a preload that needs to be integrated into the DM concept. Hereby, the lifetime of piezoelectric stacks is increased and bidirectional stroke is allowed. The calculation for the actuator working point is shown in paragraph 3. Figure 1 shows the mirror schematic, illustrating the technical principal of the SWAP-DM. A piezoelectric stack actuator (e) is pre-loaded by a spring (d). Between both is a pin (b), which transmits the translation to the mirror (a). The actuator bottom is fixed on an actuator module base (f) which is screwed into the support structure (c). The actuator module base (f) offers bores for the actuator wiring $(\mathrm{g})$.

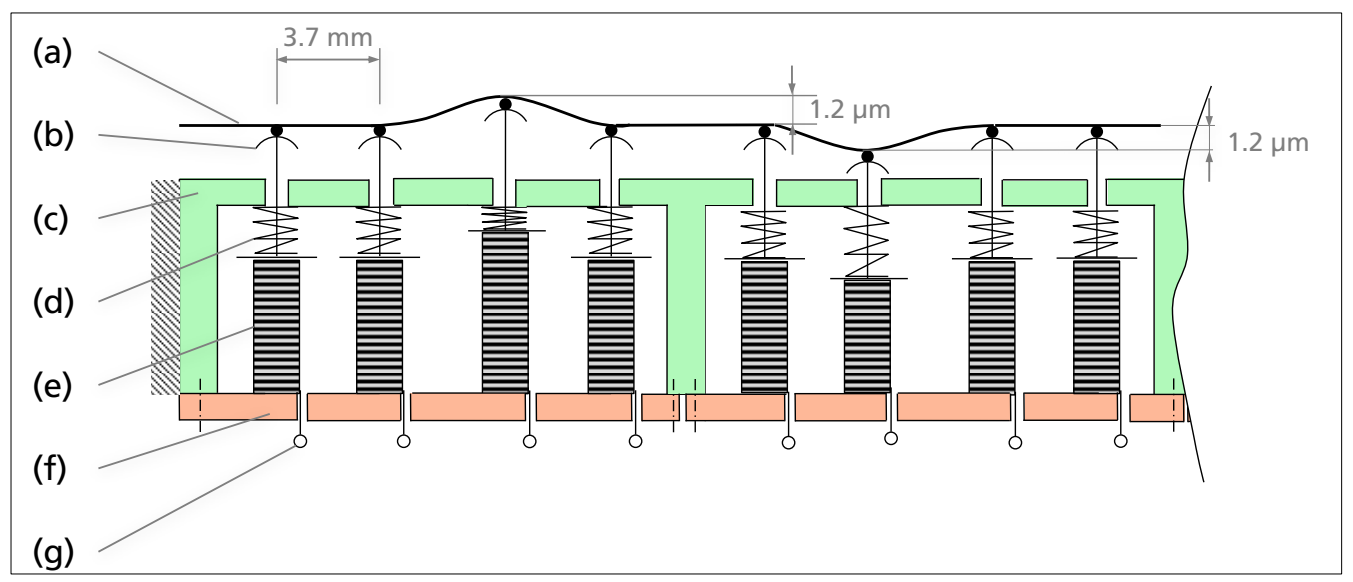

Figure 1: Schematic design of the XAO DM; (a) mirror, (b) pin, (c) support structure, (d) spring, (e) piezoelectric stack, (f) actuator module base plate, (g) wiring.

The schematic shows two times four actuators on one actuator module base plate that each form an actuator module. These modules may be removed or exchanged if necessary. In doing so, the spring (d) elongates and pulls the mirror (a) downwards to the support structure. When the new module is fixed (e.g., via screwing), the spring is compressed and introduces a pre-load on the stack actuator. Thus, bidirectional actuator movement is enabled.

In our concept, an actuator module is assembled from a module base and a number of actuators in a Cartesian grid. Each individual actuator of the module is pre-loaded by one spring. Thereby, a sufficient pre-load is established and the exchangeability of the actuators is achieved in case of failure without dismounting the mirror from its support (support structure). In case of failure, the module is removed and the connection between mirror and pin remains untouched.

The schematic concept leads to several critical technologies, which needs to be examined in several breadboards which are described in chapter 4.

Figure 2 gives the overall schematic design of the XAO SWAP DM system which is actuated by at least 688 actuator modules with 16 actuators each. Every module has one circuit board which is electrically connected by FPC/ZIF cables to 360 adapter circuit boards. The cables are about $0.5 \mathrm{~m}$ long. The adapter circuit boards are connected via $360 \mathrm{IDE}$ ribbon cable to 18 racks. These 18 racks are positioned in two towers. The ribbon cables are foreseen to be $10 \mathrm{~m}$ long to 
bridge a distance between the towers and the DM in order to exclude thermal interplay between electronics and actuators.

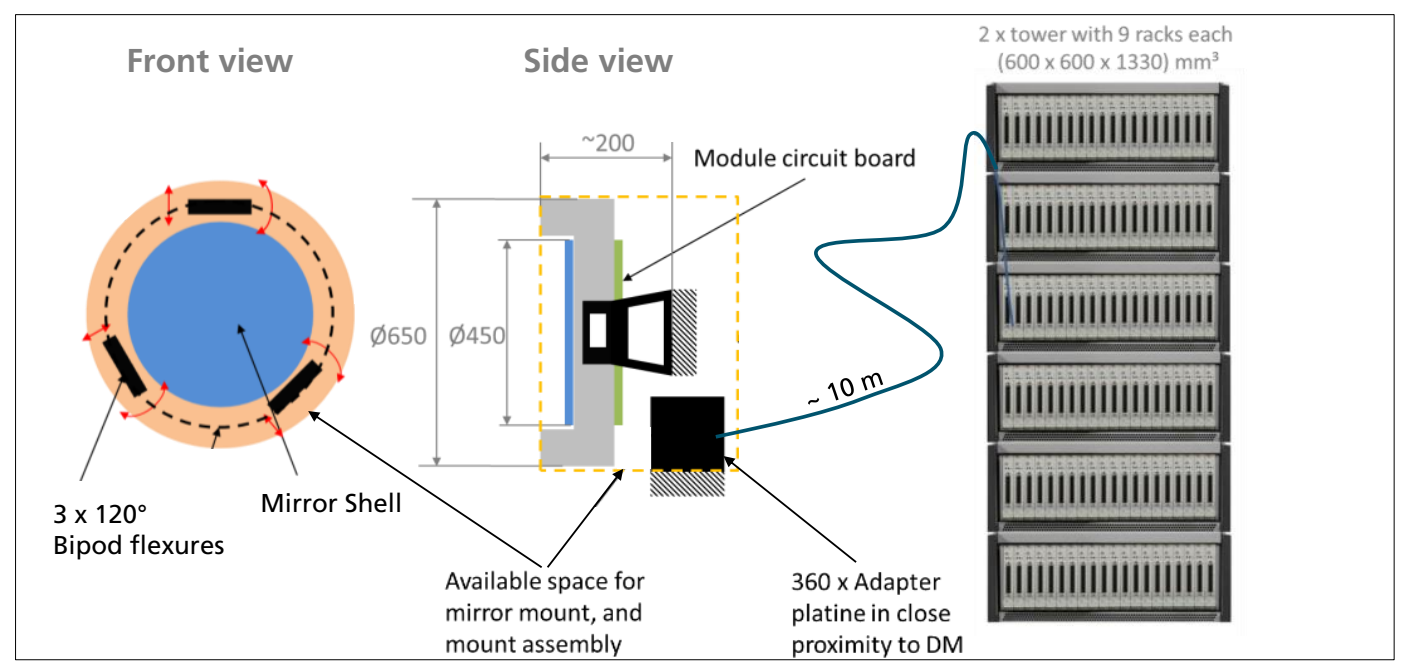

Figure 2: Overall schematic design of the XAO-System.

\section{PRELIMINARY DESIGN}

Choosing a suitable number of actuators per module is essential for the successful transfer of the preliminary design into a mechanical design. Also, the number of actuators per module defines the number of modules, which need to be installed in the final assembly. Figure 3 shows the correlation of the numbers of actuator modules over the actuators per module. With an increasing module size fewer modules need to be installed. Larger modules also lead to larger forces which act on the actuator module base plate and its fixation as all actuators from one module share the same base plate.

Besides, each module needs a support structure around on which the actuator module base is fixed. Additionally, the wall thickness of the support structure needs to be thick enough to be threaded.

Figure 3 shows another aspect of the choice of an optimal module size. The figure compares three different module sizes from $3 \times 3$ actuators to $5 \times 5$ actuators per module regarding the remaining wall thickness and the resulting acentric loading on the actuators.

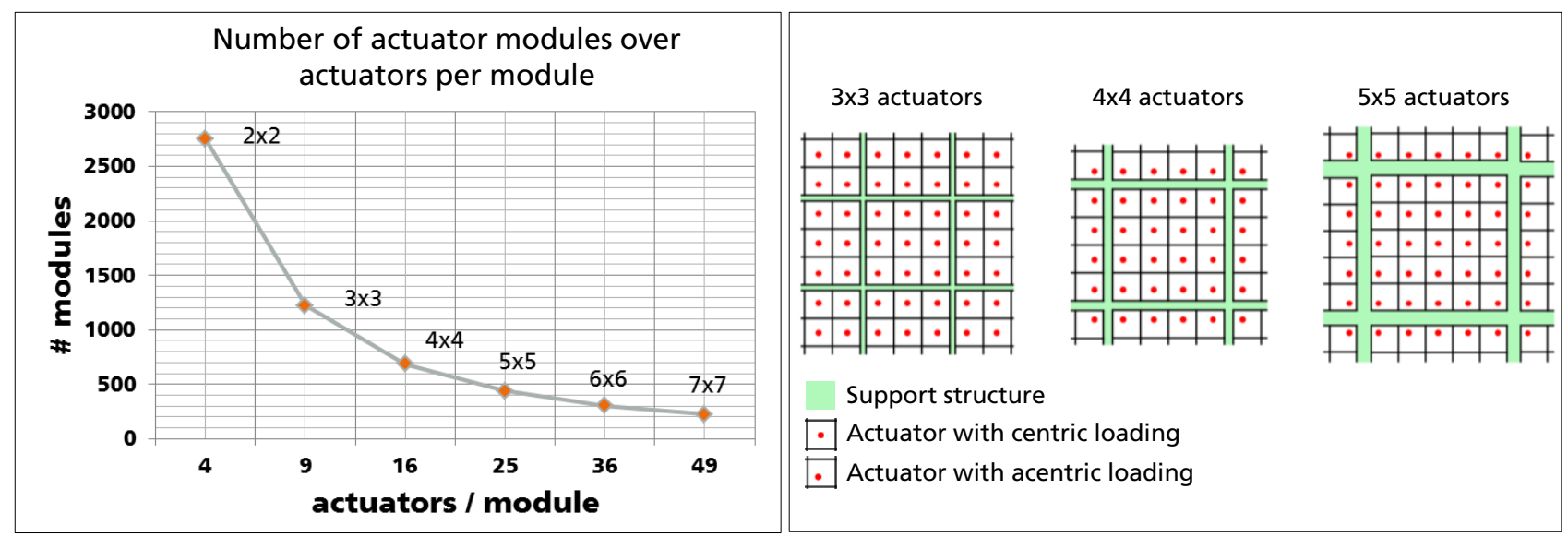

Figure 3: Number of actuator modules over the number of actuators per module (left); comparison of module sizes regarding wall thickness and acentric loading. 
The required pitch of $3.7 \mathrm{~mm}$ requires small actuator foot prints as piezoelectric stack actuators would need some space in between actuators to prevent any mechanical contact between actuators or with module walls. Contact may harm the thin insulation layer, or influences the actuator's free stroke. The actuator footprint is assumed to be $2.8 \times 3.3 \mathrm{~mm}^{2}$. In order to achieve the specified pitch, the pin may not act centrally but acentrically. The degree of acentricity increases with an increase of the module size, which is illustrated in Figure 3. Thus, we consider a module size with $4 \mathrm{x} 4$ actuators a good design value. The acentricity in such a module is below $60 \%$. This load case is examined in a test breadboard to ensure that this does not limit the actuator's lifetime.

For the preliminary design we further calculated the working point of the PICMA ${ }^{\circledR}$ stack actuator [3] for the given boundary conditions. The diagram in Figure 4 shows the calculation principle.

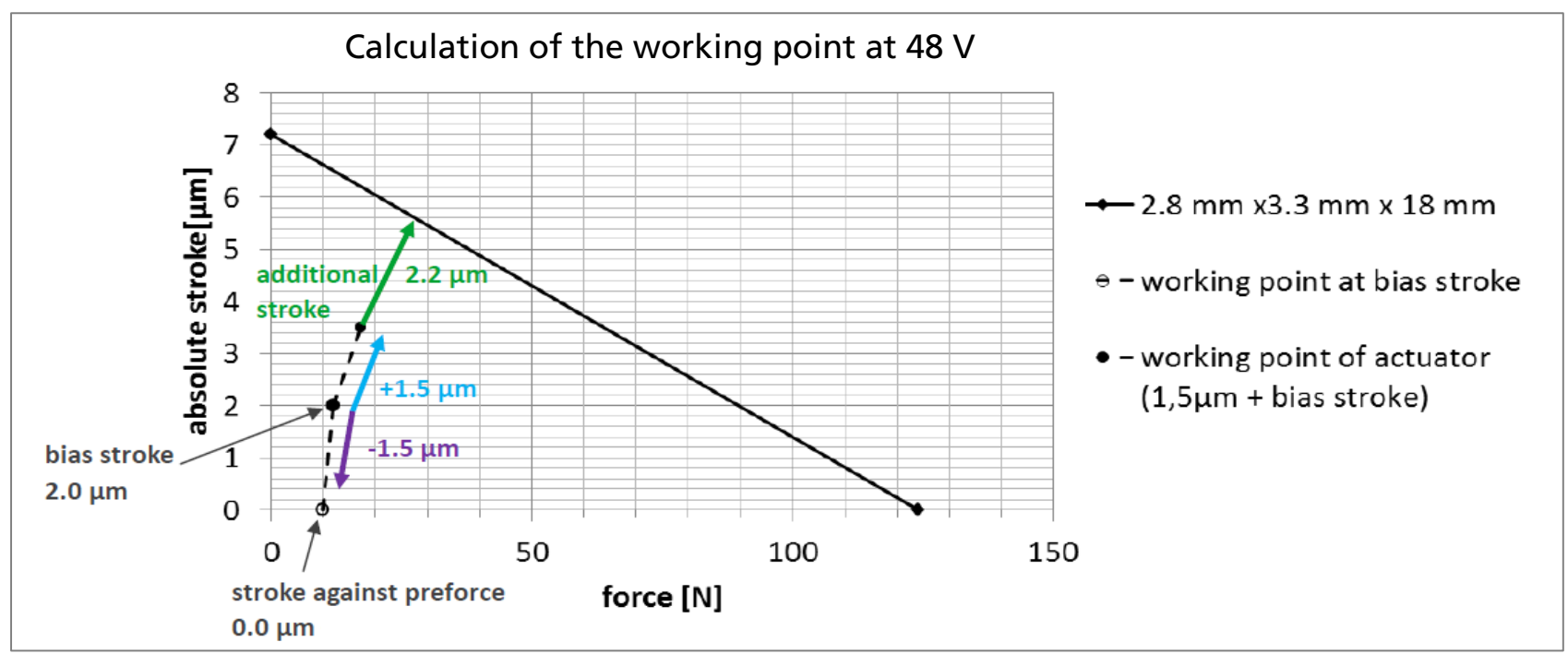

Figure 4: Calculation of the working point at $48 \mathrm{~V}$ for a PICMA ${ }^{\circledR}$ stack actuator of $2.8 \times 3.3 \times 18 \mathrm{~mm}^{3}$.

The working range of the PICMA ${ }^{\circledR}$ stack actuator of $2.8 \times 3.3 \times 18 \mathrm{~mm}^{3}$ is illustrated by the black curve, which connects the maximum possible stroke without preforce with its maximum force at zero stroke. These characteristics come from the dimensions and the material of the actuator. The stroke against the preforce is marked at $0.0 \mu \mathrm{m} / 11 \mathrm{~N}$. The bias stroke at $2.0 \mu \mathrm{m}$ marks the point, from which the positive as well as the negative stroke of $1.5 \mu \mathrm{m}$ is possible. The additional $2.2 \mu \mathrm{m}$ may be used for the compensation of the remaining manufacturing inaccuracies in order to flatten the mirror surface.

\section{BREADBOARDS}

Based on the concept for the SWAP-DM, six breadboards are planned to examine different critical technologies of the mirror development.

Breadboard (A) deals with the manufacturing of the actuator modules including the actuator stack alignment and the assembly of the modules. Breadboard (B) includes a lifetime test of the PICMA ${ }^{\circledR}$ stack regarding the lifetime cycles with, both, centric and acentric loading. Breadboard $(\mathrm{C})$ is carried out to qualify a suitable adhesive for the bonding between the pin and the mirror shell. Breadboard (D) contains the evaluation of the gluing-induced mirror shell deformations. Breadboard (E) deals with the electrical contacting and control of the piezoelectric actuators. In Breadboard (F) a demonstrator is manufactured to validate the opto-mechanical functionality of the mirror concept. Thus, we will evaluate the wavefront compensation capability of the deformable mirror in static and dynamic conditions. Breadboard (F) also includes the manufacturing process of the support structure, in which three by three actuator modules can be mounted. Therewith, we may validate also the manufacturing of the support structure that is exeptional critical because of the high required accuracies. This demonstrator is additionally used to qualify a procedure to mount around 688 actuator modules and demonstrate the exchangeability of these. 


\section{SUMMARY AND OUTLOOK}

In this paper, we presented the technical principle and preliminary design of the SWAP-DM, which contains 11,000 actuators. We developed a modular activation concept, which allows for the flexible adaptation to build large multiactuator deformable mirrors. Further, we discussed the critical aspects of the concept and presented the breadboards, which serve to examine manufacturing technologies and develop processes for the assembly and alignment of the DM. The demonstrator will be tested in the first quarter of 2018 .

\section{REFERENCES}

[1] Kaspar, M., Beuzit, J.-L., et al., "EPICS: the exoplanet imager for the E-ELT”, Proc. SPIE, 7735, (2010).

[2] Stroebele, S.,Vernet, E., et al., "Deformable mirrors development program at ESO, Proc. SPIE, 9909 (2016).

[3] www.piceramic.com/en/piezo-technology/picma, PI Ceramic GmbH, (access 10/05/2017).

\section{AKNOWLEDGEMENTS}

The work reported in this article was funded by ESO under contract number 67840/ESO/15/69547/OSZ. 